\title{
Aufwachsen in überwachten Umgebungen: Medienethische Überlegungen zum Kinderrecht auf Privatheit im Zeitalter des Digitalen
}

Ingrid Stapf

\section{Abstract}

Mit dem Aufkommen überwachungsbasierter Medientechnologien von Smart Toys, Babysitter-Kameras in Teddybären bis hin zu Sprachassistenzsystemen wie Alexa, individualisierter Lernsoftware, Tracking-Apps oder Videoüberwachung in der Kita, ist die Frage aufgeworfen, was Privatheit von Kindern heute ausmacht: Bedarf es bei Kindern anderer Konzepte als bei Erwachsenen? Wie können sie den Schutz ihrer Daten im Altersverlauf steuern lernen? Und wer sind hierfür Verantwortungsträger? Der Beitrag untersucht aus medienethischer Perspektive das Kinderrecht auf Privatheit im Zeitalter des Digitalen. Von einem Beispielfall ausgehend wird erarbeitet, was die erhöhte Verletzlichkeit von Kindern begründet, wie das Kinderrecht auf Privatheit mit Blick auf personale Selbstbestimmung umgesetzt werden kann, was den Wert von Privatheit für Kinder ausmacht sowie was daraus für ein Recht auf Privatheit in digitalen Kontexten folgt. Hierzu wird eine kinderrechtliche Perspektive eingenommen, indem Kinder als handelnde Subjekte verstanden werden, die im Zuge ihrer sich noch entwickelnden Fähigkeiten verbriefte Rechte auf Schutz, Befähigung und Beteiligung haben. Um das Zusammenspiel dieser Rechte zu ergründen werden aktuelle empirische Daten und Rahmenbedingungen aufgegriffen und argumentiert, dass die Rechte von Kindern auch im Digitalen gelten und besser durchgesetzt werden sollten, dass sie dabei aber mit Blick auf die konkrete Lebenswelt relational und kontextbezogen aufzugreifen sind. Kindliche Rechte auf Privatheit bedürfen aus menschenrechtlicher Sicht folglich pädagogischer, technischer und regulatorischer Bedingungen dafür, dass Kinder sie kennen, konkret erfahren und in der Folge aktiv selbst ausüben lernen. 


\section{Mediatisierte Kindheit und Privatheit-einführende medienethische Überlegungen}

In einem Interview mit einer 4.-Klässlerin berichtet ein Mädchen von einer Puppe, der sie im Vertrauen ihre Geheimnisse erzählt hatte. Und wie sie sich schämte, als sie erst später erfuhr, dass die Puppe alle Gespräche aufgezeichnet und gespeichert hatte. Es seien doch ibre Geheimnisse gewesen und jetzt wisse sie gar nicht, wer sie alles hören könne. ${ }^{1}$

Beispiele wie diese legen nahe, dass sich die Frage der Privatheit mit Blick auf Kinder anders als bei Erwachsenen stellt, da Kindheit eine besonders verletzliche Entwicklungsphase ist. Ausgehend von diesem Beispiel soll im Folgenden eine medienethische Perspektive auf die Frage der Privatheit von Kindern ${ }^{2}$ in überwachten Umgebungen erarbeitet werden, die in aktuelle Kontexte und Problemlagen rund um mediatisierte Kindheit eingebettet wird. Denn durch das Aufkommen überwachungsbasierter Medientechnologien, wie Smart Dolls, Babysitter-Kameras in Teddybären bis hin zu Home-Robotern wie Alexa, individualisierte Lernsoftware, Tracking-Apps oder Videoüberwachung in der Kita, ist heutige Kindheit in besonderem Maße von privatheitsgefährdenden Techniken betroffen. Was können wir dabei über den Wunsch von Kindern nach Privatsphäre wissen? Bedarf es bei Kindern anderer Konzepte als bei Erwachsenen? Und wie können Kinder den Schutz ihrer Daten steuern lernen? Und was verändert daran vielleicht auch Kindheit selbst?

Kindheit ist heute mediatisierte Kindheit (Krotz 2001, Tillmann/Hugger 2014). Dass Medien Einzug in das heutige Leben von Kindern halten, legen empirische Daten offen. Sie zeigen eine stärkere Verfügbarkeit und wachsende Nutzungszahlen von Medien bei immer jüngeren Kindern. So stellt die aktuelle KIM-Studie (MPFS 2019a) fest, dass fast alle Kinder zwischen sechs und 13 Jahren (98\%) zuhause das Internet nutzen können und die Hälfte der Kinder ein eigenes Smartphone hat. Sie recherchieren über Suchmaschinen (65\%), verschicken WhatsApp-Nachrichten (62\%) oder

1 Das Interview mit einer 4.-Klässlerin an einer Berliner Grundschule entstand im Rahmen eines medienpädagogischen Projekts von Martin Riemer und wurde auf dem Netzfest der re:publica 2019 diskutiert (vgl. https://19.netzfest.de/de/session/w o-stimmen-kindern-netzpolitik-was-es-gibt-was-es-braucht).

2 Den Begriff „Kinder“ benutze ich hier mit der UN-Kinderrechtskonvention, die Kindheit als Phase zwischen der Geburt und bis zum vollendeten 18. Lebensjahr (und de facto bis zur Volljährigkeit) versteht. Damit wird im Folgenden nicht zwischen Kindern und Jugendlichen unterschieden, es sei denn, die Begriffe werden beispielsweise in Gesetzestexten wie dem "Jugendmedienschutz" verwendet. 
schauen YouTube-Videos (56\%). Beliebteste soziale Medien der 14- bis 24Jährigen sind laut DIVSI-Studie (DIVSI 2018) WhatsApp, YouTube und Instagram. Heranwachsende verabreden sich über soziale Medien oder verhandeln Identitätsfragen über ihre Postings auf Snapchat oder Instagram. Schüler*innen recherchieren Hausaufgaben im Internet und schauen sich YouTube-Erklärvideos an. Sie sitzen in ihren Kinderzimmern und spielen vernetzte Computerspiele mit Freunden. All dies hat sich in der Folge des Digitalisierungsschubs im Zuge der Corona-Pandemie verstärkt.

Gerade weil Kinder digitale Medien schon stückweise selbst bestimmt nutzen, können sie aber auch viel früher schon Erfahrungen machen, die nicht altersgerecht oder gar verstörend sind. Mit dem ersten internetfähigen Smartphone haben Kinder Zugang zum globalen Netz, das derzeit weitgehend unreguliert ist. So gibt jeder fünfte Jugendliche an, dass schon einmal falsche oder beleidigende Inhalte über die eigene Person online oder über das Smartphone verbreitet wurden (MPFS 2019b: 49). Ein stringenter Jugendmedienschutz kann nicht mehr, wie früher, durch eine Eingangskontrolle im Kino, erreicht werden. So haben Anbieter von Pornographie wie YouPorn ihren Sitz im Ausland und können nicht ausreichend national reguliert werden. Dies hat beispielsweise zur Folge, dass Kinder pornographische Inhalte über Pornhub im Instagram-Feed abonnieren können. Und dass sie dabei auswertbare Spuren im Netz hinterlassen. Mit der „Mediatisierung sozialer Welten“ (Krotz et al. 2014) lassen sich analoge und digitale Lebenswelten nicht mehr trennscharf voneinander unterscheiden. Das betrifft Kinder wie Erwachsene gleichermaßen. In der gelebten Praxis ihrer Lebenswelt sind beide Bereiche - gerade mit Blick auf die Spuren ihrer Daten - ineinander verwoben.

Aktuell zeigt sich vor allem eine große Verunsicherung. Mediale und öffentliche Diskurse fokussieren schnell auf extreme Beispiele (wie Computerspielesucht oder „digitale Demenz“) und verbreiten „Moralpanik“. Auf diese folgen oft strikte Verbote oder Rückzugsforderungen. Viele Eltern fühlen sich überlastet und orientieren sich selbst noch in diesen Veränderungen. Die Medienforschung und die Medienregulierung kommen mit der Geschwindigkeit der Entwicklungen kaum mit. Im Bereich digitale Medien und Kindheit liegt damit ein großer gesellschaftlicher Orientierungsbedarf.

Dies ist nicht zu unterschätzen, wenn man bedenkt, dass, laut einer UNICEF-Studie, ein Drittel der weltweiten Internetnutzer*innen mittlerweile Kinder unter 18 Jahren sind (Livingstone/Carr/Byrne 2016: 7): „An estimated one in three of all Internet users in the world today is below the age of 18." Kinder sind also medial mittendrin. 
Dass Kindheit heute mediatisiert ist, meint dabei nicht nur einen technischen, sondern auch einen kulturellen und sozialen Wandel ihrer Lebenswelt. Diese ist eben auch eine datafizierte Lebenswelt, in der alle kindlichen Zugriffe, Tätigkeiten und Kontakte in der Welt der Codes und von Big Data abgebildet und ausgewertet werden. Aktuell gefährdet auch Online-Überwachung kindliche Privatheit, indem Massenüberwachungstechnologien von Regierungen und Firmen eingesetzt werden können, „to track, store, and analyse children's actions with a level of detail previously unattainable“ (Brown/Pecora 2014).

All diese Themenaspekte betreffen grundlegende Fragen der Ethik. Auch wenn es bereits erste ethische Auseinandersetzungen mit mediatisierter Kindheit und Jugend (Stapf/Prinzing/Köberer 2019) gibt, so wurde die Frage nach Privatheit mit Blick auf Heranwachsende im deutschsprachigen Raum bislang kaum wissenschaftlich bearbeitet. Heutige Kindheit ist nicht nur "mediatisierte Kindheit“ (Tillman/Hugger 2014), sondern auch datafizierte Kindheit (vgl. Lupton/Williamson 2017). Ob in der Schule oder im Kindergarten, in der Familie oder in der Freizeit: Kinder wachsen heute in zunehmend überwachten Umgebungen auf, in denen Daten über sie gesammelt, ausgewertet, Profile erstellt und damit Sichtweisen auf sie manifestiert werden, die ihre Zukunft über die Gegenwart hinaus betreffen (Stapf 2020).

Je differenzierter dabei die Techniken selbst, ihre Vernetzung untereinander, vor allem aber ihre kommerzielle Auswertbarkeit werden, desto stärker kann die Privatheit von Kindern über neue Formen der Datensammlung und Überwachung durch Unternehmen, Eltern und Staat, aber auch Schulen, bedroht werden. „Die Rechte von Kindern in digitalen Handlungswelten“, so Stapf et al. (2020: 3), sollten in der Folge „stärker durchgesetzt und berücksichtigt werden. Dazu gehören explizit das Recht auf informationelle Selbstbestimmung, der Datenschutz, die freie Entfaltung der Persönlichkeit und ein geschützter Privatbereich.“

Mit Blick auf Daten entstehen in diesen Kontexten asymmetrische Machtverhältnisse (Rieger 2013), die besonders für jüngere Kinder schwer einschätzbar sind: „The complexity of the current digital ecology makes it particularly hard, for children and adults alike to anticipate the long-term consequences of growing up in the digital age" (Stoilova et al. 2019: 4).

Ethisch bedeutsam sind diese Entwicklungen vor allem mit Blick auf demokratische Freiheitsrechte, welche individuelle Selbstbestimmung und subjektives Wohlergehen aktuell, aber auch nachhaltig ermöglichen sollen. Medien- und Informationsethik, verstanden mit Heesen (2016: 3), erfasst die Auseinandersetzung „mit der Bewertung und Steuerung individuellen, gesellschaftlichen und institutionellen Handelns für eine sozialver- 
trägliche Gestaltung von Informations- und Kommunikationstechniken wie auch mit der Verantwortung des und der Einzelnen bei ihrer Entwicklung, Verbreitung und Anwendung." Gerade mit Blick auf Kindheit als besonders verletzliche Lebensphase lassen sich vielfältige Fragen nach der Verantwortung für die Fürsorge gegenüber Kindern, aber auch ihrer Befähigung und gar Beteiligung stellen.

Diese Aspekte markieren die Grundidee auch einer kinderrechtlichen Perspektive. Ausgehend vom Eingangsbeispiel wird diese, nun folgend, mit Blick auf ein Kinderrecht auf Privatheit im Zeitalter des Digitalen medienethisch untersucht.

\section{Was macht die Besonderheit von Kindheit mit Blick auf Privatheit aus?}

Das Eingangsbeispiel zeigt erstens, dass Kinder anders in der Welt sind als die meisten Erwachsenen. Kindheit ist eine verletzliche Entwicklungsphase, in der sich viele Fähigkeiten und Fertigkeiten erst noch ausbilden, in der Kinder abhängig von für sie Sorgetragenden sind; und auch noch nicht über die Vielfalt an Erfahrungen und Informationen verfügen, die es braucht, um selbstbestimmt entscheiden zu können (vgl. Stapf 2018, 2019a, 2020).

Diese besondere Verletzlichkeit von Kindern ist der Entwicklungsdimension geschuldet, indem Kindheit eine Phase biologischer - und damit psychischer, kognitiver und physischer - Entwicklung ist. Die kindliche Vulnerabilität gründet darin, dass Kinder sich hinsichtlich ihrer kognitiven Voraussetzungen von Erwachsenen unterscheiden, dass sie weniger Vorwissen und Erfahrungen zu bestimmten gesellschaftlichen Prozessen haben sowie, dass sie, auch in der Folge davon, eine für ihre Altersgruppe spezifische Herangehensweise an Medien pflegen (Stapf et al. 2020: 7).

Berücksichtigt man diese Entwicklungsprozesse im Kindheitsverlauf, so zeigt sich die Schwierigkeit, „allgemein“ über „Kinder“ zu sprechen, da es große Unterschiede nicht nur zwischen Entwicklungsphasen (wie der frühen Kindheit und der Adoleszenz), sondern auch geschlechtliche sowie individuelle Unterschiede gibt. So ist von sensiblen Phasen ${ }^{3}$ im Kindheitsverlauf ebenso auszugehen wie von persönlichkeitsbezogenen Besonderheiten und genetische Anlagen. Hieraus folgt, dass zwar über allgemeine Aspekte

3 In der Entwicklungspsychologie werden sensible Perioden als ,jene Entwicklungsabschnitte definiert, in denen spezifische Erfahrungen maximale Wirkung haben (= Perioden höchster Plastizität). Sensible Phasen sind Zeitabschnitte, in denen spezifische Lernerfahrungen maximale Wirkung zeigen, wobei viele sensible Phasen auch durch Stadien der Hirnreifung bedingt sind“ (Stangl 2020). 
von Kindheit gesprochen werden kann, dabei aber gleichzeitig ausreichend differenziert und eine Diversität von Kindheit(en) berücksichtigt werden sollte.

Abseits biologischer und entwicklungspsychologische Aspekte verweist der Kulturhistoriker Philippe Ariès (2003) darauf, dass der Begriff Kindheit historisch und sozial immer schon im Wandel war (vgl. Ammicht Quinn in diesem Band). Auch die neuere Kindheitsforschung (vgl. Prout/James 1997 ) versteht Kindheit als ein soziales und kulturelles Konstrukt, das Erfahrungen von und Sichtweisen auf Kindheit immer auch erst (unterschiedlich) herstellt. Kindheit ist damit einerseits eine biologische Entwicklungsphase und basiert andererseits auf einem kulturell fundierten sozialen Konstrukt, aus dem heraus Sichtweisen auf Kinder erfolgen. Diese kulturell variierenden Sichtweisen definieren dann auch, was an Kindheit "schützenswert" ist oder was eine gute und gelingende Kindheit ausmacht.

Entwicklungspsychologisch betrachtet haben Erfahrungen in der Kindheit teils langfristige Auswirkungen über die Kindheit hinaus ins Erwachsenenalter. Welche (auch problematischen) Erfahrungen Kinder machen und welche grundlegenden Informationen, Fürsorgeangebote und Bewältigungsstrategien ihnen zur Verfügung stehen, ist demnach mitentscheidend für die überhaupt möglich werdende Zukunft von Kindern, d. h. für ihre Handlungs- und Gestaltungsmöglichkeiten als Erwachsene. Aus diesem Spannungsfeld folgert der Philosoph Joel Feinberg (1980) das Recht auf eine offene Zukunft. Als sich noch entwickelnde Personen bilden Kinder demnach bestimmte Fähigkeiten und Erfahrungen erst noch aus. So implizieren die Grundfreiheiten für Kinder - anders als für Erwachsene nicht nur die Abwesenheit externer Hindernisse (indem Kinder beispielsweise eine Schulpflicht haben); Einschränkungen in kindliche Freiheiten werden vielmehr dadurch gerechtfertigt, was sie eigentlich ermöglichen sollen - nämlich „the sense of being a person, meaning being responsible, having reasons, acting with intentions and purposes. We want a society that enhances the liberty of a person to develop (creatively) in the fullest sense possible" (ebd. 199).

Um eine offene Zukunft zu gewährleisten, ist folglich schon während der Kindheit die Ermöglichung eigener, informierter Entscheidungen des Kindes zentral. Und da Autonomie auch ein oberstes Bildungsziel in Demokratien ist, sollten Kinder diese Entscheidungen möglichst selbstbestimmt treffen lernen.

Wird in der Ethik also vom ,informed consent“ gesprochen - dass erst dann autonome Entscheidungen getroffen werden können, wenn das dazu verfügbare Wissen und die relevanten Informationen vorliegen und in der Folge informiert eingewilligt werden kann -, dann gilt es zu berücksichti- 
gen, dass eine informierte Einwilligung sich für Kinder anders gestaltet als für Erwachsene. Um eine informierte Einwilligung zu ermöglichen, müsste die Information kindgerecht erfolgen und es müssten Überlegungen vorausgehen, was (bei wem) vorausgesetzt werden kann und welche Unterstützung möglicherweise notwendig wird. Genau dies variiert im Entwicklungsverlauf, vor allem aber von Kind $z u$ Kind: Was hierzu notwendig wird, hängt ab von kognitiven und emotionalen Fähigkeiten, situativen und kulturellen Kontexten, dem Bildungshintergrund der Familie sowie auch Persönlichkeitsfaktoren.

So unterscheiden sich nicht nur der Umgang von vier- und 14-jährigen mit Smart Toys, sondern möglicherweise auch das Wissen darum, was dabei an Daten erhoben wird und wie Privatsphäre-Einstellungen verändert werden können. Zudem unterscheidet sich, welche Rolle Privatheit überhaupt für die einzelne Person spielt oder spielen kann. Damit Fragen der Privatheit also überhaupt entscheidungsrelevant werden, brauchen Kinder für sie nachvollziehbare Informationen, um dann - soweit möglich und sinnvoll - selbst ihre Einwilligung geben zu können.

Abbildung 1: Kontexte der Privatheit (Livingstone et al. 2019: 16)

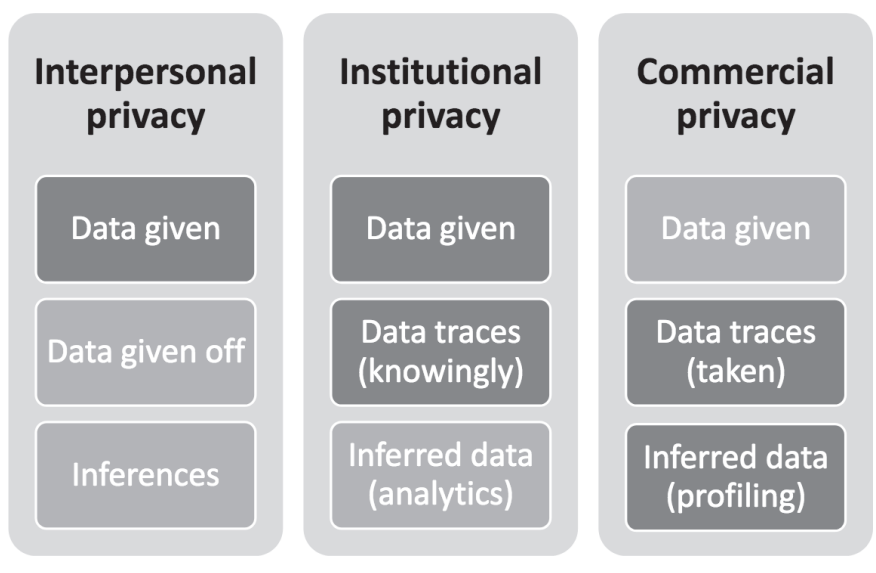

Eine aktuelle Studie von Sonia Livingstone zeigt, dass Kinder beim Thema Privatsphäre nur gering informiert sind und zunächst sehr stark vertrauen, da sie Privatheit primär als interpersonal verstehen (Stoilova/Livingstone/ Nandagiri 2019: 3, 17, 24). Sie wollen bestimmte Informationen oder Bilder beispielsweise eher vor anderen Kindern oder den eigenen Eltern schützen als vor Firmen, die sie kommerziell auswerten. Selbst Unterneh- 
men wie Instagram oder Snapchat verstehen Kinder in ihrer Wahrnehmung oft wie Personen:

„[...] children give considerable thought to interpersonal privacy, although they may struggle with how to negotiate sharing or withholding personal information in networked contexts which demand they trade privacy for opportunities for participation, self-expression and belonging" (Livingstone in diesem Band).

In der Folge achten sie weniger stark auf institutionelle Kontexte wie ihre Schule oder gar auf kommerzielle Kontexte wie das Interesse globaler Firmen an ihren Daten. Die Preisgabe ihrer Daten erfolgt dabei hauptsächlich, wenn Kinder aktiv über Interaktionen auf ihren sozialen Medien Fotos, Daten oder andere Informationen mit anderen Nutzenden austauschen. Neben dem Risiko, „dass Gleichaltrige diese zum Beispiel für Cybermobbing-Angriffe nutzen (horizontale Privatheitsbedrohung), ist auch die passive Sammlung, Analyse und der Verkauf von Daten durch Unternehmen eine Gefahr, der sich Kinder und Jugendliche nicht vollständig bewusst sind (vertikale Privatheitsbedrohung).“(Stapf et al. 2020: 4)

Während Kinder also bei den verschiedensten Anwendungen, die digital konfiguriert sind, ständig Spuren hinterlassen, haben sie vor allem in der frühen Kindheit keine Vorstellung von ihrem "growing digital footprint" (Stoilova et al. 2019: 21). Genau dies wäre aber die Bedingung dafür, dass ihr Recht auf Privatheit eine Rolle auch mit Blick auf kindliche Selbstbestimmung spielen könnte.

\section{Wie lassen sich Rechte von Kindern auf Privatheit umsetzen?}

Das Eingangsbeispiel zeigt zweitens, dass Kinder moralische Gefühle und Bedürfnisse schon früh artikulieren (vgl. Eisenberg 1992) und durchaus auch hier wieder entwicklungsabhängig - wissen, was sie fair und unfair, gerecht oder ungerecht finden. Kinder wünschen sich, auch wenn sie es vielleicht noch nicht genau so benennen, Räume für sich, d.h. für ibre eigene Privatheit. Obwohl sie selbst noch aushandeln, was Privatheit für sie bedeutet, belegt auch die Studie von Livingstone, dass ,children care about their privacy online, and they want to be able to decide what information is shared and with whom" (Stoilova/Livingstone/Nandagiri 2019: 3). Grundlegende Fairness fordern Kinder auch über die Altersspannen hinweg von Unternehmen: „companies should explain better, improve services, be age-appropriate, provide options that users want, respond to users' concerns, and treat users well” (Livingstone in diesem Band). 
Privatheit ${ }^{4}$ fungiert allgemein als „Sammelbegriff für bestimmte zu schützende Positionen“ und umfasst verschiedene Teilaspekte und auch Rechte, wie das Recht auf informationelle Selbstbestimmung, das Recht auf Schutz des Privaten und das Recht auf Datenschutz sowie das Persönlichkeitsrecht, das Post- und Fernmeldegeheimnis, die Unverletzlichkeit der Wohnung und das sogenannte IT-Grundrecht, das die Vertraulichkeit und Integrität informationstechnischer Systeme zum Schutzzweck hat (Stapf et al. 2020: 4). Privatheit hat unterschiedliche Bezugsdimensionen: Diese reichen von körperlichen Zonen, mentalen Vorgängen, über persönliche Entscheidungen, lokale Räume, den Schutz privater Daten bis hin zu institutionellen Bereichen, die sich zwar analytisch unterscheiden lassen, die aber - gerade bei Kindern - in der Praxis zutiefst ineinander verwoben sind; denn Privatheit tritt „relational innerhalb sozialer Konstellationen“ auf (Ochs 2019: 15).

Gerade in „mediatisierten Welten“ (Krotz/Hepp 2012) erleben Kinder interaktive Techniken aus ihrer konkreten Lebenswelt heraus. So titelte der Tagesspiegel im August 2019, dass viele Kinder Siri als ihre beste Freundin bezeichneten. ${ }^{5}$ Dies verwundert nicht, da Kinder bei den wichtigsten Tätigkeiten das Thema Freundschaft an oberster Stelle nennen: 93 Prozent der sechs bis 13-jährigen Kinder interessieren sich, laut KIM-Studie (MPFS 2019a: 5), für das Thema Freunde und Freundschaft. ${ }^{6}$ Es spielt für Kinder also eine zentrale Rolle bei der Nutzung digitaler Medien, es prägt aber auch ihre Wahrnehmung von digitalen Anwendungen. Denn fast alle Kinder haben in Deutschland aktuell Zugang zu Fernsehen, Internet und Smartphone (MPFS 2019a: 9).

4 Im vorliegenden Text wird vorwiegend der Begriff der Privatheit verwendet, der, anders als der eher „paternalistische Ansatz der Bestimmung eines schützenswerten Bereiches von außen („Privatsphäre“)“ durch staatliche Behörden, Justiz oder Rechtswissenschaften, die Selbstbestimmung des Einzelnen zum Maßstab erhebt (vgl. Stapf et al. 2020: 4, vgl. auch Geminn/Roßnagel 2015, Nebel 2015). Der Begriff Privatheit ist damit besser mit einem freiheitsorientierten Ansatz kompatibel. Der Begriff Privatsphäre wird im Text vor allem dann verwendet, wenn er sich auf diese Begriffsverwendung in bestimmten Dokumenten bezieht, z.B. der UN-Kinderrechtskonvention.

5 Quelle: https://www.tagesspiegel.de/wirtschaft/abhoererin-von-sprachassistent-viele -kinder-bezeichnen-siri-als-ihre-beste-freundin/24878764.html [Zugriff am 30.12.2019].

6 Gut zwei Drittel zeigen weiterhin Interesse an den Themen „Sport“, „Handy/ Smartphone“ sowie „Schule“. Gut drei von fünf Kindern begeistern sich für „Internet/Computer/Laptop“, „Musik“ und „Computer-/Konsolen-/Onlinespiele“. 
Digitale Technologien sind damit in unterschiedlichster Gestalt mit kindlichen Lebenswelten verwoben, vor allem indem sie eine wichtige Rolle für die kindliche Beziehungspflege spielen. Sie werden evident in der Kommunikation mit anderen Kindern, so bei vernetzten Computerspielen, der Nutzung von Social Media oder im Spiel mit Smart Toys. Sie strukturieren aber auch die kindliche Kommunikation mit Erwachsenen, $\mathrm{ob}$ in Familienchats oder in Bildungseinrichtungen. Und dass Kinder nicht mehr - wie nur eine Generation zuvor - zwischen „analogen“ und "digitalen" Lebenswelten unterscheiden, wirkt sich auf die (schwieriger abschätzbaren und auch kontrollierbaren) Konsequenzen ihres Handelns aus, indem sie nämlich - anders als in klassisch analogen Handlungskontexten - vermehrt Datenspuren hinterlassen.

Um diese Fragen verhandeln zu können, bedarf es einer kinderrechtlichen Perspektive. Denn selbst bestimmen zu dürfen, welche Räume andere betreten oder welche Informationen sie einsehen oder verwenden dürfen, gilt nicht als ein „Luxusgut“, sondern ein für Demokratien wesentliches Freiheitsrecht. Folgerichtig ist auch in Artikel 16 der UN-Kinderrechtskonvention $(\mathrm{UN}-\mathrm{KRK})^{7}$ verbrieft, dass

„kein Kind [...] willkürlichen oder rechtswidrigen Eingriffen in sein Privatleben, seine Familie, seine Wohnung oder seinen Schriftverkehr oder rechtswidrigen Beeinträchtigungen seiner Ehre und seines Rufes ausgesetzt werden" (darf).

Aus Sicht der Kinderrechte sind Fragen der Privatheit damit auf das Wohlergehen von Kindern ausgelegt und mit weiteren kindlichen Grundrechten, z.B. auf Bildung, Schutz und Partizipation, verknüpft. So bedarf ein umgesetztes Recht auf Privatheit Bildungsmaßnahmen darüber, wie diese gesteuert und definiert werden kann und da Kinder noch besonders verletzlich sind, folgt auch ein erhöhter Schutzbedarf. Und soll langfristig daraus ebenfalls Selbstschutz als autonomes Handeln erfolgen, ist Partizipation in all diesen Prozessen wesentlich. Die Förderung des Wohlergehens umfasst damit auch das Ziel, Kindern selbstbestimmtes Handeln, faire Chancen und wichtige Fähigkeiten für ihr Leben zu eröffnen. Hierzu versteht Art. 3 UN-KRK das Kindeswohl oder das beste Interesse (englisch „best interest“) von Kindern als übergeordnet. Es wird als eine Art „Querschnittsrecht“ gesetzt, das sich auf alle anderen Kinderrechte bezieht, und an dem sich alle auf das Kind bezogene Verfahren und Maßnahmen ausrichten sollen:

7 Online abrufbar unter: https:/www.bmfsfj.de/blob/jump/93140/uebereinkommenueber-die-rechte-des-kindes-data.pdf [Zugriff: 30.3.2020]. 
„Bei allen Maßnahmen, die Kinder betreffen, gleichviel ob sie von öffentlichen oder privaten Einrichtungen der sozialen Fürsorge, Gerichten, Verwaltungsbehörden oder Gesetzgebungsorganen getroffen werden, ist das Wobl des Kindes ein Gesichtspunkt, der vorrangig zu berücksichtigen ist."

Auch bei einem kinderrechtlichen Ansatz werden folglich immer schon Wertannahmen vorausgesetzt. Denn Kindheitsbilder prägen sowohl im Bildungsbereich als auch im Elternhaus oder in der Medienregulierung Vorstellungen darüber, was eine gute Kindheit ausmacht und was diese ermöglicht. Gesellschaftliche Vorstellungen von einer guten Kindheit nähren sich, so Fuhs (2004: 277), aus dem zugrunde liegenden Kindheitsbild sowie der „Gesamtheit aller gesellschaftlichen Bedingungen des Kinderalters." Und, laut Bühler-Niederberger (2011: 13 ff., 42) basiert das, was Kindern in Deutschland zugetraut und zugemutet werden dürfe, auf der Vorstellung einer langen und behüteten Kindheit, die den bewahrenden Schutzgedanken betont.

Aus kinderrechtlicher Sicht sind Kinder also nicht wie in vielen philosophischen Diskursen „noch-nicht-autonome“ oder gar „Mängelwesen“, denen zentrale Fähigkeiten fehlen, sondern agieren als handelnde Subjekte. ${ }^{8}$ Kinder gelten folglich nicht nur als zukünftige Erwachsene, die einmal nämlich mit 18 - autonom sein werden, sondern die bereits als Kinder Rechte haben und schon während ihrer Kindheit mit- und selbst bestimmen dürfen und damit nicht nur Objekt der Entscheidungen Sorgeberechtigter sind. ${ }^{9}$ Der kinderrechtliche Rahmen strebt „,children 's civil, political, protection, social economic and cultural rights" an: Diese sehen, so Third et al. (2019: 376), "children as active agents in the exercise of their rights" und leiten „the particular rights of children to ensure they develop to their full potential“ daraus ab. Ähnlich betont Maywald (2012: 16), dass Kinderrechte weder „Erwachsenenrechte“ noch „Sonderrechte“ für Kinder sind, sondern dass Kinder einen „auf ihre spezielle Situation zugeschnittenen Menschenrechtsschutz benötigen“. Dabei ist der Schutz ihrer Privat-

8 Für einen Überblick der philosophischen Diskussion um Kinderrechte vgl. Archard 2016, Stapf 2019a.

9 In der philosophischen Diskussion existieren verschiedene Modelle, die Kindern entweder gar keine oder nur teilweise Rechte zuschreiben. Insgesamt ist das Kindheitsbild dabei aber vermehrt auf Kindheit als Transitorium zum Erwachsenenstadium bezogen, wobei Erwachsenen per se Autonomierechte zugeschrieben werden und Kinder als nicht oder begrenzt autonomiefähig gelten (vgl. Stapf 2018, 2019, 2020). 
sphäre als wesentlich zu verstehen. Oder: für diesen Menschenrechtsschutz ist der Schutz ihrer Privatsphäre wesentlich.

\section{Ist Privatheit ein Zustand oder Teil des Prozesses sich entwickelnder Autonomie?}

Ein Großteil der philosophischen Autonomietheorien versteht Individuen erst dann als autonomiefähig, wenn bestimmte Fähigkeiten (wie rationales Handeln, Abwägen und Urteilen) bereits vorliegen. Daran haben vor allem die feministische Ethik und die Care-Ethik (vgl. Conradi 2001) kritisiert, dass dies wichtige gesellschaftliche Gruppen per se von Autonomiefähigkeit ausschließen würde, wie beispielsweise Menschen mit starken Behinderungen oder eben auch Kinder. Problematisiert wird überdies, dass unter diesen Bedingungen auch viele Erwachsene nicht (immer) autonomiefähig wären.

Versteht man Individuen dagegen als ,inherently social beings“ (Friedman 2000: 217f.), die sich lebenslang in Entwicklungsprozessen befinden, dann entfaltet sich personale Autonomie im Zuge sich entwickelnder Fähigkeiten und im Rahmen von Beziehungen. Um sie auszubilden werden zur Verfügung stehende Informationen und Wahlmöglichkeiten, wachsende Erfahrungen zu verschiedenen Formen innerer und äußerer Autonomie sowie Einsicht in mögliche Konsequenzen des eigenen Handelns notwendig. Kinder als handelnde Subjekte wahrzunehmen, obwohl sie noch nicht über alle (voran kognitiven) Fähigkeiten verfügen, die Autonomiefähigkeit begründen, wird mit relationalen Selbstbestimmungskonzepten möglich. ${ }^{10}$ Relationale Autonomiekonzepte (vgl. Mackenzie/Stoljar 2000) postulieren, dass sich Prozesse der Autonomieentwicklung im Rahmen von sozialen Beziehungen und in konkreten Kontexten ereignen. Privatheit kann also sowohl im Lebensverlauf als auch in verschiedenen Kontexten und Kulturen oder in verschiedenen Beziehungen ganz Unterschiedliches bedeuten.

10 Selbstbestimmung wird verstanden als ein Selbstverhältnis, ein Weltverhältnis oder als eine interaktionistische Beziehung (Seidel 2016). Nach internalistischen Theorien hängt Autonomie von internen Bedingungen wie mentalen Zuständen, dem geistigen Vermögen oder ausgebildeten Fähigkeiten ab. Konträr dazu sind für externalistische Theorien externe Bedingungen, wie Freiheit von Zwang, soziale Umstände oder Möglichkeiten relevant für personale Autonomie. Interaktionalistische Theorien verstehen Selbstbestimmung dagegen als ein Sich-in-BeziehungSetzen, das in einem Zusammenspiel von Selbst- und Weltverhältnis möglich wird. 
Als eine Art kontextuelle Integrität hängt sie auch von Beziehungen und Kontexten ab. Sie ist auf etwas und auf andere Personen bezogen, und damit nicht nur individuell zu verstehen (Solove 2015, Hargreaves 2017). Über die eigene Privatheit selbst bestimmen zu können impliziert damit die Möglichkeit, die eigene Privatheit auch bewusst einzuschränken, um andere Ziele zu erreichen oder Privatheit unter bestimmten Umständen gar nicht schützen zu wollen. ${ }^{11}$

Kindliches Handeln ist folglich auch Teil von sozialen Aushandlungsprozessen im Rahmen der eigenen Persönlichkeitsentwicklung, die sich im Verlauf des Lebens vollzieht. Als Persönlichkeit wird „das einem Menschen spezifische Gefüge von Merkmalen, Eigenschaften, Einstellungen und Handlungskompetenzen bezeichnet, das sich auf der Grundlage der biologischen Ausstattung und als Ergebnis der Bewältigung von Lebensaufgaben ergibt“; damit gilt Persönlichkeitsentwicklung auch als „die Veränderung wesentlicher Elemente dieses Gefüges im Verlauf des Lebens“ (Bründel/Hurrelmann 2017: 16).

Ein Kind muss also nicht schon über alle Fähigkeiten verfügen, um selbstbestimmt zu handeln. Ganz im Gegenteil braucht es zur Ausbildung dieser Fähigkeiten entsprechende Erfahrungen. So zeigt das Eingangsbeispiel drittens, dass Kinder in ihren konkreten Lebenswelten schon weitgehend selbstbestimmt handeln. Das Mädchen hat mit der Puppe gespielt, ohne dass sie wusste, dass ihre Gespräche gespeichert werden. Sie hat ihre Daten nicht fahrlässig aus der Hand gegeben, sondern es geschah, weil es ihr nicht transparent war, was in diesem Fall der Unterschied zwischen einer Puppe mit und ohne Informationstechnik war. Noch jüngere Kinder dagegen wären vielleicht noch nicht in der Lage, eine Folgenabschätzung zu leisten, selbst wenn sie wüssten, dass die Daten abgehört werden können. Das Gleiche könnte aber auch auf technisch nicht sehr kompetente oder daran nicht interessierte Erwachsene zutreffen.

11 Das Phänomen scheinbar widersprüchlichen Verhaltens durch die freiwillige Aufgabe von Privatheit wird in der Forschung als „privacy paradox“ (Barnes 2006, Norberg/Horne/Horne 2007) bezeichnet und kritisch diskutiert (Baruh/Secinti/ Cemalcilar 2017). Vermutet wird, dass Kinder im Internet einerseits freiwillig persönliche Informationen online teilen und dabei Risiken für ihre Sicherheit und ihre Privatheit in Kauf nehmen, obwohl sie andererseits ihre Privatheit schützen wollen. Der Widerspruch besteht mit Blick auf soziale Kontexte allerdings eigentlich darin, dass soziale Teilhabe nur bei Aufgabe herkömmlicher Privatheitsvorstellungen möglich wird. Kinder müssten hierbei, wie Erwachsene auch, eine wirkliche Wahl haben (White Paper Kinderrechte und Privatheit vgl. Stapf et al. 2020: 5). 
Die Frage ist also nicht, $o b$ Kinder ein Recht auf Privatheit im Digitalen haben, sondern, wie ibnen Bedingungen geschaffen werden können, durch die sie ihre Privatheit erleben und erfahren können. Und wie sie ihre Privatheit selbst bestimmen und regulieren lernen können. Dies erfordert aus kinderrechtlicher Sicht aber geradezu, dass Kinder nicht nur als handelnde Subjekte gesehen, sondern auch als solche behandelt werden.

Blickt man auf Beispiele des Sharenting, wenn Eltern teils intime Fotos ihrer noch Ungeborenen oder ihrer Babys auf sozialen Netzwerken posten, so zeigt sich, dass vor allem jüngere Kinder oft als „Extension des Selbst“ wahrgenommen werden und weniger als Subjekte oder gar Rechteträger. Phänomene des elterlichen Sharenting als „habitual use of social media to share news, images, etc. of one's children" 12 und Oversharenting, wenn dies exzessiv geschieht, verdeutlichen, dass es mit Blick auf Selbstbestimmungsrechte von Kindern offensichtliche Widersprüche gibt (Stapf 2018): So wird die Social-Media-Nutzung von WhatsApp von Kindern durch Eltern begrenzt und ist rechtlich zum Schutz der Kinder gar auf das Alter von 16 Jahren angehoben worden, ${ }^{13}$ während

„parents share information about their children online, they do so without their children's consent. These parents act as both gatekeepers of their children's personal information and as narrators of their children's personal stories [...]. A conflict of interests exists as children might one day resent the disclosures made years earlier by their parents.“ (Steinberg 2017: 839)

Spy-Apps von Eltern oder andere digitale Tools zum Überwachen kindlicher Aktivitäten in der Schule können zwar dem Schutz des Kindes dienen und folgen auch aus dem verbrieften Kinderrecht (Art. 5 UN-KRK) zur elterlichen Fürsorgepflicht: Diese steht im Zusammenspiel - und oft im Konflikt - mit kindlichen Rechten, soll dabei aber ermöglichen, „das Kind bei der Ausübung der in diesem Übereinkommen anerkannten Rechte in einer seiner Entwicklung entsprechenden Weise angemessen zu leiten und zu führen." Werden elterliche Fürsorgepflichten vom Kind her gedacht, dann folgen aus der Frage nach Privatheit andere, kritische Nachfragen. Dann lässt sich beispielsweise hinterfragen, ob Maßnahmen, wie Spy-Apps,

12 vgl. Collins Dictionary „Sharenting“, online unter: https://www.collinsdictionary.com/dictionary/english/sharenting [Zugriff: 1.4.2020].

13 So war das Mindestalter von 13 Jahren auf 16 Jahre angehoben worden und wird im Zuge der neuen Datenschutz-Grundverordnung jetzt auch von Kindern abgefragt (vgl. https:/www.faz.net/aktuell/wirtschaft/unternehmen/whatsapp-setzt-mi ndestalter-auf-16-jahre-herauf-15558790.html [Zugriff: 17.12.2019]). 
dazu führen können, dass Kinder sich als verantwortliche Subjekte erfahren und sich selbst schützen lernen oder ob Kinder diese Maßnahmen eher als Überwachung erleben, denen sie passiv ausgeliefert sind.

\section{Was macht den Wert der Privatheit für Kinder aus?}

An dem Eingangsbeispiel zeigt sich viertens, warum Privatheit in freiheitlichen Demokratien überhaupt so einen hohen Wert hat und mit grundlegenden demokratischen Freiheitsrechten verknüpft ist. Privacy umfasst, so Westin (1967), die individuelle Kontrolle über Informationen, die wissentlich gegeben oder mit anderen geteilt werden. Sie gilt, nach Nissenbaum (2010: 3), als „a right to appropriate flow of personal information“.

Privatheit wird generell Erwachsenen zugeschrieben, bei Kindern treten dagegen grundlegende Fragen zutage: Haben beispielsweise schon Neugeborene, die noch gewickelt werden, ein Recht auf eine Privatsphäre? Wie können wir Personen eine Privatsphäre zuerkennen, die sie selbst noch nicht als solche wahrnehmen, artikulieren oder gar einfordern können? Wie bereits beschrieben gibt es zu diesem moralischen Problem unterschiedliche ethische Positionen, die sich auf unterschiedliche Autonomiekonzepte beziehen. Aus einer menschenrechtlichen Perspektive heraus, die hier eingenommen wird, erscheint es schwierig, Bedingungen daran zu knüpfen, welche Fähigkeiten jeweils schon vorliegen müssen, damit einer Person grundlegende Rechte zugeschrieben werden oder diese selbst bestimmen oder mitbestimmen darf. Unteilbare, unkündbare und universelle Menschenrechte sind, so Bielefeld (2008), vielmehr „inklusiv“ zu denken.

Denn die Idee der Menschenrechte basiert auf dem Prinzip der Menschenwürde, die allen Menschen zukommt: Das altersübergreifende Gleichheitsprinzips (Art. 1 der Allgemeinen Erklärung der Menschenrechte; Art. 3 GG) impliziert, dass Kinder einen eigenen moralischen Status haben, der nicht Ableger des Status anderer, voran der Eltern, ist (vgl. Schickhardt 2012). Kinder haben also das fundamentale Recht, als Gleiche behandelt $\mathrm{zu}$ werden.

Versteht man Kinderrechte als „Menschenrechte für Kinder“ (Maywald 2012), dann gilt es aber gleichermaßen, wesentliche Differenzen zwischen Kindern und Erwachsenen zu berücksichtigen, d.h. dass Kinder eben noch in der Entwicklung sind. Autonomie ist für das Aufwachsen ein wichtiger Zielbegriff, da Autonomie aus menschenrechtlicher Sicht überhaupt erst die Grundlage für moralische Verantwortungsübernahme, staatsbürgerliche Partizipation in Demokratien, die Grundlage weiterer Menschenrechte, aber auch für ein individuelles gelingendes Leben ist. 
Autonomieentwicklung verstanden als ein interaktiver Prozess, der im Zuge der „evolving capacities“ (Lansdown 2005) lebenslang wächst, erfordert damit, schon kleinsten Kindern ein Recht auf Privatsphäre zuzusprechen, auch wenn diese den Wunsch danach selbst noch nicht artikulieren können. Vielmehr rückt dann die Frage in den Vordergrund, was es braucht, damit Kinder ihre Selbstbestimmung entfalten und entfalten lernen können: Welche Prozesse und Interaktionen, welche Formen der Befähigung, des Schutzes, aber auch der Partizipation ermöglichen es Kindern, Privatheit zu erleben, sie einzufordern, für wichtig zu halten und sie selbst zu gestalten? Viele Eltern und Erziehende verfügen hier selbst nicht über alle Kompetenzen, um Kinder optimal zu begleiten. Kinder haben ihren Eltern gegenüber sogar oft Wissens- und Erfahrungsvorsprünge:

"Children are often pioneers in exploring and experimenting with new digital technologies and services [...]. Increasingly independent users of digital technologies and starting at a much younger age, children experience newly emerging risks often before adults know about their existence or are able to put mitigating strategies in place. In the contemporary digital environment, children's actions are particularly consequential as technologies transform their lives into data which can be recorded, tracked, aggregated, analysed and monetised - and which is durable, searchable and virtually undeletable." (Stoilova et al. 2019: 4)

Je jünger Kinder sind, desto verletzlicher können sie dabei noch sein. Selbst wenn sie Medien zwar schon (d.h. im deskriptiven Sinne) selbstbestimmt nutzen können, fehlen ihnen noch weitreichende Erfahrungen über mögliche Folgen ihres Handelns sowie Entscheidungskriterien, die sie moralisch gesehen (d.h. im normativen Sinne) leiten können. Beispielsweise zeigen Studien wie die von Livingstone et al. (2019), dass Kinder unter 11 Jahren in ihrer Entwicklung meist nicht weit genug vorangeschritten sind, um Konzepte wie „Privatheit“ vollumfänglich zu begreifen:

„Vor dem Hintergrund ihrer noch nicht vollständig abgeschlossenen Entwicklung sind Kinder und Jugendliche somit auch besonders anfällig für Online-Dienste, die auf kurzfristige Erfolgserlebnisse, Belohnungsanreize und soziale Honorierung setzen und im Gegenzug Datenprofile der Nutzenden sammeln" (Stapf et al. 2020: 7).

Privatheit ist folglich kein erreichbarer Zustand, sondern vielmehr relational zu verstehen (vgl. Livingstone et al. 2019). Sie ist bezogen auf den aktuellen Entwicklungsstand und die individuelle Persönlichkeit ebenso wie auf Beziehungen, Kontexte und Situationen. Gerade kindliche Praktiken hängen stark von der sozialen Umgebung ab. Somit geht es beim Schutz 
der Privatheit zwar systemisch um eine Ökologie der Daten im Netz, aber in sozialen Kontexten und der Lebenswelt geht es auch um eine gelebte Kultur der Privatsphäre (Livingstone 2019 et al., Stapf 2019b). Damit ist der Wert der Privatheit für Kinder der gleiche Wert wie für Erwachsene, er ist aber stärker im Entwicklungsgeschehen der besonders verletzlichen Lebensphase Kindheit zu denken.

Die UN-Kinderrechtskonvention von 1989 artikuliert in 54 Artikeln kindereigene Rechte ${ }_{2}$ die auf den vier Prinzipien Recht auf Gleichbehandlung, Vorrang des Kindeswohls, Recht auf Leben und Entwicklung und Achtung vor der Meinung des Kindes beruhen (Maywald 2012: 96). Die drei Säulen der Kinderrechte verbinden Schutz- (protection), Versorgungs- (provision) sowie Beteiligungsrechte (participation), die als eine Einheit zu verstehen sind, dem das beste Interesse von Kindern übergeordnet ist. Hierzu wird es notwendig, die Sichtweise und die Perspektive von Kindern auf das, was sie betrifft, angemessen einzubeziehen. ${ }^{14}$ Genau das wäre als zentraler Bestandteil einer gelebten Kultur der Privatsphäre zu verstehen und folgt wesentlich aus der Entwicklungsdimension von Kindheit.

So mag das Kind im Eingangsbeispiel erlebt haben, dass es unangenehm ist, wenn man nicht weiß, wer die eigenen Geheimnisse anhören kann. Sie konnte es der Puppe nicht ansehen, wer alles auf die Daten Zugriff hat. Die Idee eines Geheimnisses, dass es - wenn überhaupt - nur ausgewählte Personen wie die beste Freundin, die ältere Schwester, oder eben nur die Puppe, mit der man eine besondere Beziehung hat, erfahren dürfen, ließ sich in diesem Fall nicht auf ein Smart Toy übertragen. Dabei spielt auch eine Rolle, dass das Mädchen diese Situation nicht vollumfänglich erkennen oder von der Puppe oder gar den Spieleherstellern fordern konnte, dass das Geheimnis geheim bleibt. Allerdings folgt daraus nicht, dass das Mädchen aufgrund dieser fehlenden Fähigkeiten oder Optionen kein Recht auf Privatheit hätte. Vielmehr sollte Privatheit für sie - auch als Kind - greifbarer werden, indem sie ein „lebendiges“ Menschenrecht wird, das beispielsweise im Design voreingestellt ist und somit eine wirklich wählbare Option wird. Solange Kinder noch besonders verletzlich sind,

14 Der unbestimmte Rechtsbegriff des „Kindeswohls“ („wellbeing of the child“, „best interests of the child“) vereint subjektive Aspekte des Willens und der Befindlichkeit des Kindes sowie objektive kindliche Interessen. Das Kindeswohl soll subjektiv gesichert werden, indem die Sichtweise des Kindes als Betroffener in Entscheidungsprozessen, das heißt, die Kinderperspektive, angemessen berücksichtigt wird. Und es soll objektiv gefördert werden, indem gesellschaftlich definiert wird, was ein gelingendes Leben für Kinder ausmacht, das heißt, welche Bedingungen, Möglichkeiten und Kompetenzen dafür wesentlich sind. 
sollte Privatheit folglich bei digitalen Anwendungen vorkonfiguriert sein (i.S. von privacy-by-design).

\section{Was folgt für das Recht von Kindern auf Privatheit im Digitalen?}

Ein ethisches Kernproblem des Themas ist die Objektivierung der Nutzenden, die Unsichtbarkeit der Verknüpfung von Daten und deren Auswertung, sowie eine neuartige Form der Nachbaltigkeit von Datenspuren. Spuren, die Kinder im Netz hinterlassen, sind, metaphorisch gesprochen, nicht im Sand markiert, sondern in Beton gegossen (vgl. Stapf 2019b). Sie können spätere Optionen verhindern, wenn Unternehmen vor dem Vorstellungsgespräch Facebook-Einträge in Alkoholfeste in der Jugend einsehen können. Sie können zu Scham und Ohnmacht führen, wenn Kinder Cybermobbing erfahren. Oder sie können über die Erstellung von Dossiers oder das längerfristige Tracking von Kindern ihr Recht auf eine offene $\mathrm{Zu}$ kunft einschränken.

Datenspuren können aber auch individuell auf Bedürfnisse von Kindern einwirken und auf ihre besonderen Interessen oder Fähigkeiten zugeschnitten werden, wie individualisierte Lernsoftware bei Kindern mit besonderen Fähigkeiten, Einschränkungen oder gar Behinderungen. Wesentlich wird also, was auch positiv erreicht und ermöglicht werden soll und wie dies gelingen kann. Und das heißt zu reflektieren, wie aus Herausforderungen möglichst Chancen werden.

Für Kinder geht es in erster Linie um Tätigkeiten und Interessen. Aber ebenso auch um Neugier, Langeweile, Trial \& Error oder Lust auf Unterhaltung und Spiel - auch das Recht auf Freizeit und Spiel (Art. 31 UN-KRK) ist ein verbrieftes Kinderrecht. Was Chancen für ein gelingendes Leben, Aufwachsen und eine offene Zukunft ausmacht, hat folglich mit allgemein geteilten Vorstellungen von Wohlergehen ebenso zu tun wie mit subjektiven Besonderheiten. Entscheidend aus kinderrechtlicher Sicht ist die Wahrnehmung der Perspektive der Kinder selbst, die es zu berücksichtigen und die es auch sinnvoll einzubeziehen gilt. Und dazu ist es wesentlich, dass das Kinderrecht auf Privatsphäre auch im Digitalen gilt. 
Mit Blick auf das Beispiel werden abschließend ein paar Forderungen aufgestellt ${ }^{15}$ :

1. Die Privatsphäre von Kindern ist auch in digitalen Kontexten ein verbrieftes Kinderrecht. Die Kinderrechtskonvention ist seit 1992 ratifiziert und in einfaches Recht umzusetzen. Im Zuge der aktuellen Diskussion einer Aufnahme von Kinderrechten ins Grundgesetz hätte dies Auswirkungen auf unterschiedliche Lebensbereiche. Es sollte daher in digitalen Kontexten ausbuchstabiert werden, um die informationelle Selbstbestimmung schon von Kindern zu ermöglichen, wobei die besondere Verletzlichkeit von Kindern auch einen besonderen Schutz von Kindern begründet.

2. Mit der Sicherung kindlicher Privatsphäre ist das Recht des Kindes auf eine offene Zukunft verbunden. Aus Sicht der Kinderrechte sind Fragen der Privatsphäre auf das Wohlergehen von Kindern ausgelegt. Es geht darum, Kindern eine gelingende Kindheit und gute Chancen und wichtige Fähigkeiten mit Blick auf ihr Erwachsenenleben zu eröffnen. Dazu gehört das Recht auf eine offene Zukunft. Dies impliziert Sorgfalt im Umgang mit kindlichen Daten, das Recht auf Vergessen im Netz sowie auf Datensparsamkeit mit Blick auf Datenspuren, die Kinder im Netz hinterlassen.

3. Da Kinder noch in der Entwicklung sind und Fähigkeiten noch ausbilden, sollten Maßnahmen zum Schutz von Kindern immer auch Befähigungsmaßnahmen implizieren. Sowohl Schule als auch Eltern sollten Medienerziehung und Medienbildung vorantreiben, indem Kinder über ihre Rechte informiert werden und verschiedene Formen von Privatsphäre in digitalen Kontexten kennen und selbst regulieren lernen. Der DigitalPakt Schule der Bundesregierung ${ }^{16}$ sollte neben Geräten und Infrastrukturen die digitalen Kompetenzen von Lernenden wie Lehrenden in Bildungskontexten fördern mit dem Ziel, dass Kinder selbst informierte Entscheidungen treffen lernen können.

4. Anreizsysteme für Datenschutz by Design \& Default durch Unternehmen, Plattformbetreibern und Bildungseinrichtungen sollten gefördert werden. Privatheit im Digitalen sollte immer eine Möglichkeit sein und nicht erst aktiv eingestellt werden müssen. Es muss für Kinder selbst wählbar sein, in welchem Grad von Öffentlichkeit sie sich jeweils

15 Vertiefend hierzu vgl. White Paper Kinderrechte und Privatheit (Stapf et al. 2020: 16ff.).

16 Vgl. https:/www.bundesregierung.de/breg-de/themen/digital-made-in-de/digitalp akt-schule-1546598 [Zugriff: 25.7.2020]. 
befinden wollen. Und dies muss einfach im Design erkennbar sein, z.B. über auditive Ansagen oder visuelle Gestaltung („Wenn Du das abschickst, können es alle Menschen sehen, die das gleiche Angebot nutzen"). Die Privatsphäre von Kindern wird schon in der Konzeption von Angeboten, Software und Nutzungsmöglichkeiten vorkonfiguriert. Anbieter sollten in der Entwicklung, im Design und bei der Umsetzung von Angeboten Anreizsysteme hin zu "privacy-by-design“ vorfinden. Sie sollten verpflichten werden, grundlegende Standards einzuhalten.

5. Privatsphäre ist ein die Demokratie sicherndes Menschenrecht. Hierzu bedarf es angesichts der rasanten technischen Entwicklung gesamtgesellschaftlicher, interdisziplinärer und kontextsensibler Ansätze. Die Möglichkeit zu entscheiden, welche Informationen in bestimmten Kontexten oder mit bestimmten Personen geteilt werden und welche nicht, ist grundlegend für personale Autonomie und damit ein Kernthema freiheitlicher Demokratien. Zu verstehen wie Heranwachsende Privatsphäre heute im Digitalen erleben und wie sich dies im Altersverlauf entwickelt, ist eine Forschungsaufgabe. Hierzu braucht es interdisziplinäre Langzeitstudien - auch unter Beteiligung von Heranwachsenden - innovative technische Ansätze, gesellschaftliche Diskurse und einen flexiblen Kinder- und Jugendmedienschutz.

Das Eingangsbeispiel hat aufgezeigt, wie verwoben das Netz an Herausforderungen mit Blick auf die Privatheit und den Datenschutz von Kindern im Digitalen sein kann. Dabei gilt es aus ethischer Sicht immer zu differenzieren: Kindheit ist weder eine einheitliche homogene Lebensphase, wie auch digitale Medien nicht gleichförmig sind. Zweijährige lassen sich mit 17-Jährigen ebenso wenig vergleichen wie Erklärvideos der NASA mit der Smart Barbie.

Was aber dringend gesellschaftlich diskutiert werden sollte, ist, was es jeweils braucht, damit sich Potenziale entfalten und Herausforderungen mit Blick auf die Privatheit von Kindern im Digitalen minimiert werden können. Denn es geht darum, dass möglichst viele Kinder frei und verantwortlich, aber auch mit Freude selbstbestimmt handeln lernen.

\section{Literatur}

Ariès, Philippe (2003): Geschichte der Kindheit. München: dtv.

Archard, David W. (2016): Children's Rights. In: The Stanford Encyclopedia of Philosophy. In: Zalta, E. N. (Hg.). Online unter: https://plato.stanford.edu/archives/su m2016/entries/rights-children/ [Zugriff: 1.4.2020]. 
Barnes, Susan B. (2006): A privacy paradox: Social networking in the United States. In: First Monday, 11(9). https://doi.org/10.5210/fm.v11i9.1394.

Baruh, Lemi / Secinti, Ekin / Cemalcilar, Zeynep (2017): Online privacy concerns and privacy management: A meta-analytical review. In: Journal of Communication, 67(1), S. 26-53.

Bielefeldt, Heiner (2008): Menschenwürde. Der Grund der Menschenrechte. Berlin: Deutsches Institut für Menschenrechte. Online unter: https:/www.ssoar.info/ss oar/handle/document/31608.

Brown, Duncan H. / Pecora, Norma (2014): Online Data Privacy as a Children's Media Right: Toward Global Policy Principles. In: Journal of Children and Media, vol. 8, no. 2, S. 201-207.

Bründel, Heidrun / Hurrelmann, Klaus (2017): Kindheit heute. Lebenswelten der jungen Generation. Weinheim/Basel: Beltz.

Bühler-Niederberger, Doris (2011): Lebensphase Kindheit: Theoretische Ansätze, Akteure und Handlungsräume. Weinheim: Beltz.

Conradi, Elisabeth (2001): Take Care. Grundlagen einer Ethik der Achtsamkeit. Frankfurt a. M.: Campus.

Deutsches Institut für Vertrauen und Sicherheit im Internet (DIVSI) (2018): DIVSI U-25-Studie. Euphorie war gestern. Die „Generation Internet" zwischen Glück und Abhängigkeit. Hamburg.

Eisenberg, Nancy (1992): The Caring Child. Cambridge: Harvard University Press.

Feinberg, Joel (1980): A Child's Right to an Open Future. In: Aiken, W. / LaFollette, H. (Hg.), Whose Child? Parental Rights, Parental Authority and State Power. Totowa, NJ: Littlefield, Adams \& Co., S. 124-153.

Friedman, Marilyn (2000): Feminism in Ethics: Conceptions of Autonomy. In: The Cambridge Companion to Feminism in Philosophy, Cambridge: Cambridge University Press, S. 205-219.

Fuhs, Burkhard (2004): Kindheit. In: Krüger, H.-H. / Grunert, C. (Hg.): Wörterbuch Erziehungswissenschaft. Wiesbaden, S. 274-280.

Geminn, Christian / Roßnagel, Alexander (2015): „Privatheit" und, „Privatsphäre“ aus der Perspektive des Rechts - ein Überblick. In: JuristenZeitung, 70(14); S. 703-708.

Hargreaves, Stuart (2017): Relational privacy and tort. In: William and Mary Journal of Women and the Law 23(3), S. 433-476.

Heesen, Jessica (2016): Handbuch Medien- und Informationsethik. Stuttgart: Metzler.

Krotz, Friedrich / Despotović, Cathrin / Kruse, Merle-Marie (Hg.) (2014): Die Mediatisierung sozialer Welten. Synergien empirischer Forschung. Wiesbaden: VS Springer.

Krotz, Friedrich / Hepp, Andreas (2012): Mediatisierte Welten. Forschungsfelder und Beschreibungsansätze. Wiesbaden: VS Springer.

Krotz, Friedrich (2001): Die Mediatisierung kommunikativen Handels. Der Wandel von Alltag und sozialen Beziehungen, Kultur und Gesellschaft durch die Medien. Wiesbaden: Westdeutscher Verlag. 
Lansdown, Gerison (2005): The Evolving Capacities of the Child. Florenz: UNICEF.

Livingstone, Sonia / Stoilova, Mariya / Nandagiri, Rishita (2019): Children's data and privacy online: Growing up in a digital age. An evidence review. London: London School of Economics and Political Science.

Livingstone, Sonia / Carr, John / Byrne, Jasmina (2016): One in Three: Internet Governance and Children's Rights. Florenz: UNICEF Innocenti.

Lupton, Deborah / Williamson, Ben (2017): The datafied child: the dataveillance of children and implications for their rights. In: New Media \& Society 19(5), S. 780-794.

Mackenzie, Catriona / Stoljar, Natalie (2000): Relational autonomy: feminist perspectives on autonomy, agency, and the social self. New York: Oxford University Press.

Maywald, Jörg (2012): Kinder haben Rechte! Kinderrechte kennen - umsetzen - wahren. Weinheim/Basel: Beltz/Juventa.

Medienpädagogischer Forschungsverbund Südwest (MPFS) (2019a): KIM 2018. Kindheit - Internet - Medien. Basisuntersuchung zum Medienumgang 6-13-Jähriger in Deutschland. Stuttgart.

Medienpädagogischer Forschungsverbund Südwest (MPFS) (2019b): JIM 2019. Jugend - Information - Medien. Basisuntersuchung zum Medienumgang 12-19-Jähriger in Deutschland. Stuttgart.

Nebel, Maxi (2015): Schutz der Persönlichkeit - Privatheit oder Selbstbestimmung. Verfas- sungsrechtliche Zielsetzungen im deutschen und europäischen Recht. Zeitschrift für Datenschutz, S. 517-522.

Nissenbaum, Helen (2010): Privacy in Context. Technology, Policy, and the Integrity of Social Life. Stanford: Stanford University Press.

Norberg, Patricia A./Horne, David/Horne, Dan (2007): The privacy paradox: Personal information disclosure intentions versus behaviors. Journal of consumer affairs, 41(1), S. 100-126.

Ochs, Carsten (2019): Teilnabmebeschränkungen und Erfahrungsspielräume: Eine negative Akteur-Netzwerk-Theorie der Privatheit. In: Behrendt, H. / Loh, W. / Matzner, T. / Misselhorn, C. (Hg.): Privatsphäre 4.0. Eine Neuverortung des Privaten im Zeitalter des Digitalen. Berlin: Metzler, S. 13-31.

Prout, Alan / James, Allison (1997): A New Paradigm for the Sociology of Childhood? Provenance, Promise and Problems. In: James, A. / Prout, A. (Hg.), Constructing and Reconstructing Childhood. London: Falmer Press, S. 7-34.

Rieger, Frank (2013): Von Daten und Macht. In: Aus Politik und Zeitgeschehen. 63. Jahrgang; 15-16-2013; S. 3-7: Online verfügbar unter: https://www.bpb.de/apuz/1 57536/transparenz-und-privatsphaere [Zugriff: 1.4.2020].

Schickhardt, Christoph (2012): Kinderethik. Der moralische Status und die Rechte der Kinder. Münster: Mentis.

Seidel, Christian (2016): Selbst bestimmen: Eine philosophische Untersuchung personaler Autonomie. Berlin: De Gruyter.

Solove, Daniel J. (2015): The meaning and value of privacy. In: Roessler, B. I Mokrosinska, D. (Hg.). Social dimensions of privacy: interdisciplinary perspectives. Cambridge: Cambridge University Press. 
Stangl, Werner (2020): Stichwort: 'sensible Perioden'. In: Online Lexikon für Psychologie und Pädagogik. Online verfügbar unter: https://lexikon.stangl.eu/1523/sen sible-perioden-phasen/ [Zugriff: 26.5.2020].

Stapf, Ingrid / Prinzing, Marlis / Köberer, Nina (Hg.) (2019): Aufwachsen mit Medien. Zur Ethik mediatisierter Kindheit und Jugend. Baden-Baden: Nomos.

Stapf, Ingrid / Judith Meinert / Jessica Heesen / Nicole Krämer / Regina Ammicht Quinn / Felix Bieker / Michael Friedewald / Christian Geminn / Nicholas Martin / Maxi Nebel / Carsten Ochs (2020): Privatheit und Kinderrechte, White Paper Forum Privatheit. Schriftenreihe: Forum Privatheit und selbstbestimmtes Leben in der digitalen Welt, Creative Commons 2020. Online verfügbar unter: https://www.forum-privatheit.de/publikationen/white-paper-policy-paper/.

Stapf, Ingrid (2020): Kindliche Selbstbestimmung in digitalen Kontexten - medienethische Überlegungen zur Privatsphäre von Heranwachsenden. In: Buck, F. / Drerup, J. I Schweiger, G. (Hg.): Neue Technologien - neue Kindheiten? Ethische und bildungsphilosophische Perspektiven. Stuttgart: J.B. Metzler, S. 31-54.

Stapf, Ingrid (2019a): Zwischen Selbstbestimmung, Fürsorge und Befäbigung. Kinderrechte im Zeitalter mediatisierten Heranwachsens. In: Stapf, I. / Prinzing, M. / Köberer, N. (Hg.): Aufwachsen mit Medien. Zur Ethik mediatisierter Kindheit und Jugend. Nomos, 2019, S. 69-84.

Stapf, Ingrid (2019b): „Ich sehe was, was Du auch siehst.“ Wie wir die Privatsphäre von Kindern im Netz neu denken sollten und was Kinder möglicherweise dabei stärkt ein kinderrechtlicher Impuls. In: frühe Kindheit 2-19, S. 12-25.

Stapf, Ingrid (2018): Kindliche Selbstbestimmung in der digital vernetzten Welt: Kinderrechte zwischen Schutz, Befähigung und Partizipation mit Blick auf ,evolving capacities“. In: merzWissenschaft Kinder|Medien|Rechte - Komplexe Anforderungen an Zugang, Schutz und Teilhabe im Medienalltag Heranwachsender. München: kopaed, S. 7-18.

Steinberg, Stacey (2017): Sharenting: Children's Privacy in the Age of Social Media (March 8, 2016). 66 Emory Law Journal 839. University of Florida Levin College of Law Research Paper No. 16-41.

Stoilova, Mariya / Livingstone, Sonia / Nandagiri, Rishita (2019): Children's data and privacy online: Growing up in a digital age. Research findings. London: London School of Economics and Political Science.

Third, Amanda / Livingstone, Sonia / Lansdown, Gerison (2019): Recognizing children's rights in relation to digital technologies: Challenges of voice and evidence, principle and practice. In: Wagner, B. / Kettermann, M. C. / Vieth, K. (Hg.): Research Handbook of Human Rights and Digital Technology. Cheltenham, UK \& Northhampton MA, USA: Edward Elgar, S. 376-410.

Tillmann, Angela / Hugger, Kai-Uwe (2014): Mediatisierte Kindheit - Aufwachsen in mediatisierten Lebenswelten. In: Friedrichs, H. / Junge, T. / Sander, U. (Hg.): Jugendmedienschutz in Deutschland. Wiesbaden: VS Verlag-Verlag, S. 31-45.

Westin, Alan F. (1967): Privacy and Freedom. New York: Atheneum. 
